\title{
The impact of acute remote ischaemic preconditioning on cerebrovascular function
}

\author{
Howard H. Carter ${ }^{1,2} \cdot$ Joseph D. Maxwell ${ }^{2}$. Ylva Hellsten ${ }^{1} \cdot$ Andrew Thompson ${ }^{3} \cdot$ Dick H. J. Thijssen ${ }^{2,4} \cdot$ Helen Jones $^{2}$
}

Received: 13 May 2019 / Accepted: 30 December 2019 / Published online: 13 January 2020

(c) The Author(s) 2020

\begin{abstract}
Purpose Remote ischaemic preconditioning (RIPC) refers to the protection conferred to tissues and organs via brief periods of ischaemia in a remote vascular territory, including the brain. Recent studies in humans report that RIPC provides neuroprotection against recurrent (ischaemic) stroke. To better understand the ability of RIPC to improve brain health, the present study explored the potential for RIPC to acutely improve cerebrovascular function.

Methods Eleven young healthy (females $n=6$, age; $28.1 \pm 3.7$ years) and 9 older individuals (females $n=4$, age $52.5 \pm 6.7$ years) at increased risk for stroke (cardiovascular disease risk factors) underwent assessments of cerebrovascular function, assessed by carbon dioxide $\left(\mathrm{CO}_{2}\right)$ reactivity and cerebral autoregulation during normo- and hypercapnia $\left(5 \% \mathrm{CO}_{2}\right)$ following 40 mins of bilateral arm RIPC or a sham condition. Squat-to-stand manoeuvres were performed to induce changes in blood pressure to assess cerebral autoregulation $(0.10 \mathrm{~Hz})$ and analysed via transfer function.

Results We found no change in middle cerebral artery velocity or blood pressure across 40 mins of RIPC. Application of RIPC resulted in no change in $\mathrm{CO}_{2}$ reactivity slopes (sham vs RIPC, $1.97 \pm 0.88$ vs $2.06 \pm 0.69 \mathrm{~cm} / \mathrm{s} / \mathrm{mmHg} P=0.61$ ) or parameters of cerebral autoregulation during normocapnia (sham vs RIPC, normalised gain\%, $1.27 \pm 0.25$ vs $1.22 \pm 0.35$, $P=0.46)$.

Conclusion This study demonstrates that a single bout of RIPC does not influence cerebrovascular function acutely in healthy individuals, or those at increased cardiovascular risk. Given the previously reported protective role of RIPC on stroke recurrence in humans, it is possible that repeated bouts of RIPC may be necessary to impart beneficial effects on cerebrovascular function.
\end{abstract}

Keywords Remote ischaemic preconditioning $\cdot$ Cerebral autoregulation $\cdot$ Cerebral blood flow $\cdot$ Hypercapnia

Communicated by I. Mark Olfert.

Howard H. Carter and Joseph D. Maxwell: joint first authors.

Dick H. J. Thijssen and Helen Jones: joint senior.

Helen Jones

h.jones1@1jmu.ac.uk

1 Department of Nutrition, Exercise and Sports, Integrative Physiology Group, University of Copenhagen, Copenhagen, Denmark

2 Research Institute of Sport and Exercise Science, Liverpool John Moores University, Tom Reilly Building, Byrom Street, Liverpool L3 3AF, UK

3 Institute of Translational Medicine, University of Liverpool, Liverpool, UK

4 Department of Physiology, Radboud Institute of Health Sciences, Radboud University Medical Centre, Nijmegen, The Netherlands

\author{
Abbreviations \\ $\mathrm{CO}_{2} \quad$ Carbon dioxide \\ CVC Cerebrovascular conductance \\ CVD Cardiovascular disease \\ LSD Least significant difference \\ MAP Mean arterial pressure \\ MCAv Middle cerebral artery velocity \\ $\mathrm{PetCO}_{2}$ Partial pressure of carbon dioxide \\ TCD Transcranial Doppler \\ TFA Transfer function analysis \\ RIPC Remote ischaemic preconditioning
}

\section{Introduction}

Remote ischaemic preconditioning (RIPC) is a technique that offers enhanced hypoxic tolerance and protection to systemic organs and tissues following repeated brief periods of 
ischaemia and reperfusion to a remote vascular bed (Lim and Hausenloy 2012). This phenomenon, mediated via a neural and/or humoral pathway (Shimizu et al. 2009; Jensen et al. 2012), was first described in canine hearts (Przyklenk et al. 1993) with subsequent studies demonstrating its efficacy in humans. More specifically, RIPC has been reported to reduce cardiovascular events in patients following coronary artery bypass and percutaneous coronary intervention surgeries (Thielmann et al. 2013; Davies et al. 2013), and reduce brachial artery endothelial ischemia reperfusion damage (Kharbanda et al. 2002). Given these broad potent protective effects, it is possible that RIPC may also affect the brain and cerebral vasculature.

Animal studies have reported RIPC-mediated neuroprotection in the form of reduced infarct size and improved neurological recovery following prolonged cerebral ischaemia and hypothermic circulatory arrest (Jensen et al. 2011; Ren et al. 2008). Extending these findings to humans, a study in patients with aneurysmal subarachnoid haemorrhage reported 3 to 4 bouts of RIPC within 2-12 days post event induced changes indicative of cerebral vasodilation (via morphological clustering and analysis of intracranial pulse) (Gonzalez et al. 2013). A study in stroke survivors reported increased cerebral perfusion and $70 \%$ lower stroke recurrence following daily RIPC for 300 days, compared to a group of patients receiving standard care (Meng et al. 2012). This protective effect was reinforced in a recent study in acute stroke patients where repeated application of RIPC significantly improved clinical status and reduced National Institutes of Health Stroke Scale scores (England et al. 2017), while RIPC was found to significantly reduce white matter hyperintensities volume in small vessel disease patients (Wang et al. 2017). Strict regulation of brain blood flow in response to metabolic demand and stimuli such as blood pressure and arterial blood gases is crucial for the maintenance of cerebrovascular health and is impaired in numerous clinical groups, including stroke survivors. Based on previous observations that repeated RIPC improves peripheral macro- and microvascular health in humans (Kharbanda et al. 2002; Jones et al. 2014), the observed benefits of RIPC on cerebrovascular health may be related to acute improvements in cerebrovascular function in vivo. Assessing the impact of RIPC on cerebrovascular function would (1) extend our fundamental understanding of the acute effects of RIPC in humans, and (2) may provide insight into how RIPC mediates neuroprotection and further establish it as a novel therapeutic strategy in clinically vulnerable groups.

The primary aim of this proof of principle study was to assess the impact of bilateral arm RIPC on resting cerebral blood velocity and cerebral vascular function as assessed by cerebral autoregulation and cerebral vascular reactivity to carbon dioxide $\left(\mathrm{CO}_{2}\right)$ in healthy individuals, compared to a sham condition. The $\mathrm{CO}_{2}$ reactivity assessment was selected based on previous studies suggesting it is an indicator of cerebral endothelial function (Lavi et al. 2006; Hoiland et al. 2017), while dynamic cerebral autoregulation is an indicator of cerebral vascular health and impaired in patients with cardiovascular disease when compared to healthy individuals (Caldas et al. 2016). To examine the effectiveness of RIPC across a broader spectrum of vascular health, we also included participants at an increased risk for cardiovascular disease (CVD) and stroke. Finally, previous studies have reported that hypercapnia (induced by inhalation of higher concentrations of $\mathrm{CO}_{2}$ ) transiently disrupts cerebral autoregulation and has been used as a model for impaired autoregulation (Jeong et al. 2016; Panerai et al. 1999; Zhang et al. 1998; Ainslie et al. 2008). Therefore, the secondary aim of this study was to assess the ability of RIPC to attenuate hypercapnia-induced impairment of cerebral autoregulation. We hypothesised that RIPC would improve cerebral autoregulation and $\mathrm{CO}_{2}$ reactivity, while attenuating the hypercapnia-induced impairment in cerebral autoregulation, when compared to a sham condition in both young healthy individuals and those with increased cardiovascular risk.

\section{Materials and methods}

\section{Participants}

Twenty participants were recruited for the study [healthy; $n=11$ (females $n=6$ ) and CVD risk; $n=9$ (females $n=4$ ), Table 1]. Healthy young participants (age $28 \pm 4$ years) were recreationally active, engaged in low-to-moderate intensity exercise 2-3 days per week, and were free from cardiovascular diseases, including diabetes, hypertension

Table 1 Group characteristics

\begin{tabular}{lccr}
\hline Characteristics & $\begin{array}{l}\text { Healthy indi- } \\
\text { viduals, } n=11 ; \\
\text { female }=6\end{array}$ & $\begin{array}{l}\text { CVD risk indi- } \\
\text { viduals, } n=9 ; \\
\text { female }=4\end{array}$ & $P$ value \\
\hline Age (years) & $28 \pm 4$ & $53 \pm 7$ & $<0.001$ \\
Height $(\mathrm{cm})$ & $173.1 \pm 10.1$ & $169.4 \pm 10.3$ & 0.44 \\
Weight $(\mathrm{kg})$ & $71.7 \pm 13.6$ & $93.6 \pm 23.9$ & 0.02 \\
BMI $\left(\mathrm{kg} / \mathrm{m}^{2}\right)$ & $24 \pm 3$ & $32 \pm 6$ & $<0.001$ \\
MAP $(\mathrm{mmHg})$ & $89 \pm 4$ & $104 \pm 3$ & $<0.001$ \\
PetCO $(\mathrm{mmHg})$ & $37.8 \pm 2.0$ & $40.2 \pm 2.9$ & 0.10 \\
$\left.\mathrm{MCAv}_{(\mathrm{cm} \mathrm{s}}{ }^{-1}\right)$ & $70 \pm 15$ & $54 \pm 8$ & 0.02 \\
\hline
\end{tabular}

Values are means $\pm \mathrm{SD}$

$B M I$ body mass index, MAP mean arterial pressure, $\mathrm{PetCO}_{2}$ partial pressure of end tidal carbon dioxide, $M C A v$ middle cerebral artery velocity 
or hypercholesterolemia. For the second group, older individuals ( $53 \pm 7$ years) with cardiovascular risk factors were recruited based on having $\geq 1$ of the following criteria; body mass index $>30 \mathrm{~g} / \mathrm{m}^{2}$ or a waist circumference $\geq 94 \mathrm{~cm}$ (male), $\geq 80 \mathrm{~cm}$ (female), blood pressure systolic $>130$ /diastolic $>85 \mathrm{mmHg}$ or diagnosed with high cholesterol (total $>200 \mathrm{mg} / \mathrm{dL}$, triglycerides $>150 \mathrm{mg} / \mathrm{dL}$, $\mathrm{LDL}>100 \mathrm{mg} / \mathrm{dL}$ ). Smokers, individuals with angina, heart failure or a history of myocardial infarction, transient ischaemic attack or stroke and thrombosis were excluded from participation. Participants were informed of the study protocol verbally and in writing before providing written informed consent. The study was approved by the University Research Ethics Committee and adhered to the standards set out in the Declaration of Helsinki.

\section{Study design}

Participants attended the laboratory on two occasions (separated by a minimum of 3 days). All tests were performed at the same time of day to control for diurnal variation in cerebrovascular function (Ainslie et al. 2007). All participants arrived at the laboratory following an overnight fast and had refrained from alcohol, exercise and caffeine for $24 \mathrm{~h}$ prior to each visit. Visits were randomised and counterbalanced to receive either the bilateral upper arm RIPC or the sham condition. Each visit consisted of the bilateral assessment of middle cerebral blood velocity (MCAv) during RIPC or sham. Following this cerebral autoregulation was assessed using a 5 min squat-stand protocol $(0.10 \mathrm{~Hz})$. This was then proceeded by a $5 \mathrm{~min}$ rest period, followed by $4 \mathrm{~min}$ of hypercapnia $\left(5 \% \mathrm{CO}_{2}\right)$ and then another 5 min squat-stand $\left(0.10 \mathrm{~Hz}\right.$ ) protocol but whilst breathing $5 \% \mathrm{CO}_{2}$ (See Fig. 1). The phase of menstrual cycle was not controlled for in the female participants.

\section{Study procedures}

\section{Remote ischaemic preconditioning and sham}

The RIPC condition consisted of 8 bouts in total involving the inflation of a pneumatic cuff (Hokanson SC10D; USA) on the upper arm using a rapid inflator (EC-20; D.E Hokanson) to $220 \mathrm{mmHg}$ for $5 \mathrm{~min}$. This protocol was based on a previous study that revealed $4 \times 5$ min bouts of occlusion and reperfusion on alternate limbs induced a greater improvement in exercise performance compared to unilateral occlusion and reperfusion (Cocking et al. 2018). Cuffs were inflated in an alternating fashion allowing for one arm to be occluded while the contralateral arm underwent reperfusion. The sham condition consisted of the identical protocol with the difference that the cuff pressure was inflated to only $10 \mathrm{mmHg}$.

\section{Cerebral blood flow (middle cerebral artery blood velocity)}

Following $20 \mathrm{~min}$ rest in the supine position, bilateral MCAv's were continuously measured through the temporal window using transcranial Doppler ultrasonography (TCD). Two 2-MHz Doppler probes (Spencer Technologies, Seattle, USA) were adjusted until an optimal signal was identified and held in place using a Marc 600 head frame (Spencer Technologies, Seattle, USA). Once the optimal MCAv signal was attained, the probe location and machine settings (depth, gain and power) were recorded to identify the same imaging site for the second testing session. Participants were instrumented with a two-way valve mouthpiece (Hans Rudolph) from which end tidal $\mathrm{CO}_{2}\left(\mathrm{P}_{\mathrm{ET}} \mathrm{CO}_{2}\right)$ was measured using a calibrated gas analyser (ML206 ADinstruments, Colorado Springs, USA). Continuous beat-by-beat blood pressure was obtained from a digit (Finapres, Amsterdam, Netherlands) and heart rate acquired from a 3 lead electrocardiogram. All data was sampled at $50 \mathrm{~Hz}$ with the data acquisition system PowerLab via the interface LabChart 7 (ADinstruments, Colorado Springs, USA).

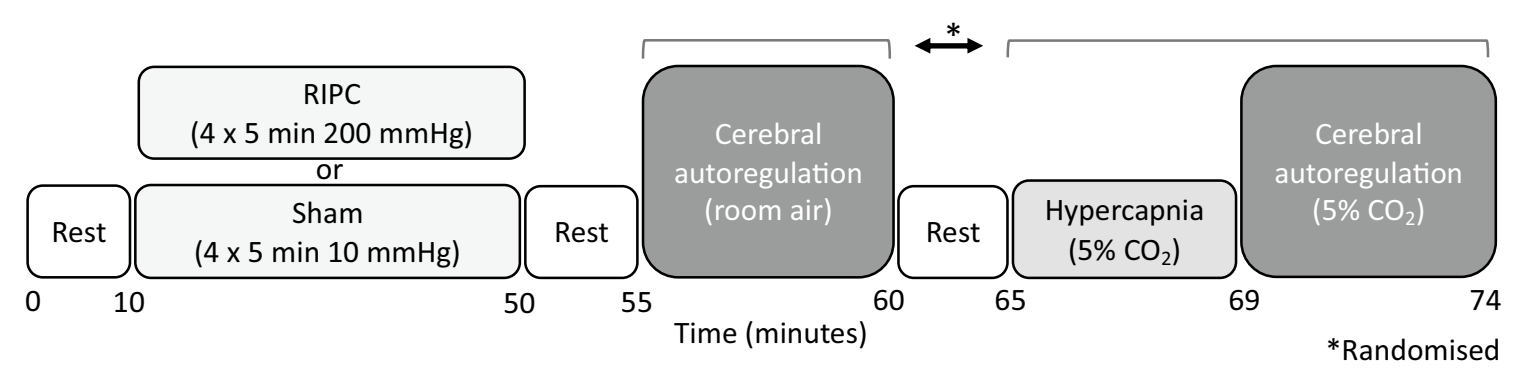

Fig. 1 Schematic of the protocol for each testing visit 


\section{Cerebral autoregulation}

Dynamic cerebral autoregulation was assessed using a squatto-stand procedure that induces transient changes in arterial blood pressure (Claassen et al. 2009; Smirl et al. 2015). Participants replicated the experimenter whilst performing the manoeuvres that involved moving from a standing upright position to squatting until the legs achieved a $90^{\circ}$ angle. Participants performed two sets at $0.10 \mathrm{~Hz}$ ( $5 \mathrm{~s}$ squat $-5 \mathrm{~s}$ stand) while breathing normal atmospheric air, and again during hypercapnia (detailed below). The first set of squat-stands was preceded by $5 \mathrm{~min}$ of seated rest while the second set immediately followed the 4 min of hypercapnia.

\section{Carbon dioxide reactivity}

Following a rest period of $5 \mathrm{~min}$, a baseline measurement of cerebral blood velocity, MAP and $\mathrm{P}_{\mathrm{ET}} \mathrm{CO}_{2}$ was performed across 2 min while participants breathed in room air. Following the baseline period, the inhaled air was switched to a Douglas bag $(100 \mathrm{~L})$ containing $5 \% \mathrm{CO}_{2}, 21 \%$ oxygen and balanced nitrogen, while participants sat in a rested seated position.

\section{Data analysis}

MCAv and MAP during the 40 min RIPC and sham conditions were averaged and extracted from LabChart in $5 \mathrm{~min}$ intervals $(n=20)$. MCA cerebrovascular conductance (CVC) was calculated as MCAv/mean arterial pressure (MAP). Calculation of the cerebrovascular $\mathrm{CO}_{2}$ reactivity slopes were performed via linear regression analysis of the two timepoints; baseline (MCAv, MAP, $\mathrm{P}_{\mathrm{ET}} \mathrm{CO}_{2}$ averaged across $2 \mathrm{~min}$ ) and $5 \% \mathrm{CO}_{2}$ (data averaged across the last $30 \mathrm{~s}$ of the 4 min hypercapnia). Two participants in the cardiovascular risk factor group were unable to complete the hypercapnic protocol, therefore data analysis for $\mathrm{CO}_{2}$ reactivity was performed on $n=18$ (Healthy =11).

Cerebral autoregulation data were extracted from LabChart beat-to-beat (MAP and MCAv) before spline interpolation and assessed via transfer function analysis (TFA) based on the Welch algorithm, using a provided script (https://www.car-net.org/). The 5 min squat-stand recordings were subdivided into five windows overlapping by $50 \%$ and passed through a Hanning window before fast Fourier transform analysis (MathWorks-Inc., Natick, Massachusetts). The cross-spectrum between MAP and MCAv was determined and divided by MAP auto-spectrum to formulate functions; normalised gain, absolute gain, phase and coherence (MAP-MCAv linearity). Gain represents the difference in amplitudes between the cerebral blood velocity and blood pressure signals, while phase describes the temporal alignment between the input (MAP) and output
(MCAv). Gain and phase data were excluded from statistical analysis if coherence was $<0.4$. TFA was performed in accordance with standardised guidelines from the Cerebral Autoregulation Research Network (Claassen et al. 2016). TFA parameters of the driven oscillations were band averaged across the very low (VLF; 0.02-0.07 Hz), low (LF; $0.07-0.2 \mathrm{~Hz}$ ) and high (HF; $0.2-0.4 \mathrm{~Hz})$ frequency domains. We induced BP oscillations at $0.10 \mathrm{~Hz}$ in the current study, this falls within the ranges of the LF domain. Therefore, the low frequency $(0.07-0.20 \mathrm{~Hz})$ output is the most appropriate to be reported as cerebral autoregulation is highly active with this frequency of squats (Zhang et al. 1998). $\mathrm{P}_{\mathrm{ET}} \mathrm{CO}_{2}$ data was averaged across each 5 min squat-stand recording. One participant in the cardiovascular risk factor group was unable to complete the cerebral autoregulation protocol during normocapnia while three participants from the same group were unable to complete the protocol during hypercapnia, therefore data was analysed on $n=18$ for the normocapnic and $n=17$ for the hypercapnic cerebral autoregulation conditions.

\section{Statistical analysis}

A three-factor group $\times$ condition $\times$ time (group; healthy vs CVD risk factors, condition: RIPC vs sham, time: 5 min intervals during intervention) general linear model was employed to analyse resting MCAv and MAP during the RIPC and sham intervention. A three-factor -capnia $\times$ group $\times$ condition (capnia; normocapnic or hypercapnic, group; healthy vs CVD risk factors, condition: RIPC vs sham) general linear model was employed to analyse the cerebral autoregulation. Hypercapnic $\mathrm{CO}_{2}$ reactivity responses were analysed via a linear mixed model and assessed for a three-way interaction (group $\times$ condition $\times \mathrm{PetCO}_{2}$ ). $\mathrm{MCAv}$ was entered as the outcome variable, with $\mathrm{PetCO}_{2}$ as a predictor variable and MAP as a covariate. $\mathrm{PetCO}_{2}$ was also entered as a random factor in the model. Statistically significant main effects and interactions were followed up with the least significant difference (LSD) approach for multiple comparisons. Statistical analysis was conducted using Statistical Package for Social Sciences (Version 22; SPSS Inc., Chicago, IL, USA). Statistical significance was delimited at $P<0.05$. Data are presented in the text as mean $(95 \%$ confidence interval) unless otherwise stated.

\section{Results}

\section{Group characteristics}

Resting MCAv was significantly higher in the healthy compared to CVD risk group (Table $1, P=0.02$ ), while resting MAP was significantly lower in the healthy compared to 
Table 2 Cerebral autoregulation analysis via transfer function using squat-stand manoeuvres

\begin{tabular}{lccl}
\hline & Healthy & CVD risk & $P$ value \\
\hline \multicolumn{2}{l}{ Sham condition-normocapnia } & & \\
PetCO $_{2}(\mathrm{mmHg})$ & $37.46 \pm 1.89$ & $40.28 \pm 3.19$ & 0.10 \\
Dynamic cerebral autoregulation $(0.10 \mathrm{~Hz})$ & & \\
MCAv power $(\mathrm{cm} / \mathrm{s})^{2}$ & $88.78 \pm 39.65$ & $64.89 \pm 80.16$ & 0.16 \\
MAP power $\left(\mathrm{mmHg}{ }^{2}\right)$ & $118.91 \pm 50.60$ & $87.01 \pm 80.16$ & 0.12 \\
Normalised gain \% & $1.25 \pm 0.30$ & $1.30 \pm 0.20$ & 0.87 \\
Gain (cm/s/mmHg) & $0.84 \pm 0.19$ & $0.74 \pm 0.12$ & 0.46 \\
Phase (radians) & $0.55 \pm 0.37$ & $0.60 \pm 0.55$ & 0.89 \\
Coherence & $0.61 \pm 0.10$ & $0.67 \pm 0.09$ & 0.12 \\
\hline
\end{tabular}

Resting comparison of healthy and CVD risk participants

Values are means \pm SD

the CVD risk group (Table $1, P=0.001$ ). For resting comparisons of cerebrovascular function between the groups, responses to the $\mathrm{CO}_{2}$ reactivity and cerebral autoregulation tests during the sham condition are reported. No difference was evident in $\mathrm{CO}_{2}$ reactivity slopes between the healthy and CVD risk groups at rest $[2.15(1.60,2.70)$ vs $1.68(1.13$, $2.24) \mathrm{cm} / \mathrm{s} / \mathrm{mmHg}, P=0.44]$, or for any of the dynamic cerebral autoregulation variables (Table 2 ).

\section{Impact of RIPC on resting cerebral blood velocity and haemodynamics}

There was no impact of RIPC on MCAv across the 40 min (Fig. 2, $P=0.58$ ). There was a group* condition interaction, with MAP being higher during RIPC compared to sham in the CVD risk group over the $40 \mathrm{~min}$ intervention period $(\mathrm{P}<0.005)$, whilst MAP was similar between conditions in the healthy group.

\section{Impact of RIPC on cerebrovascular $\mathrm{CO}_{2}$ reactivity}

The inhalation of $5 \% \mathrm{CO}_{2}$ significantly increased $\mathrm{PetCO}_{2}$ following the sham and RIPC conditions, respectively (Table 3,
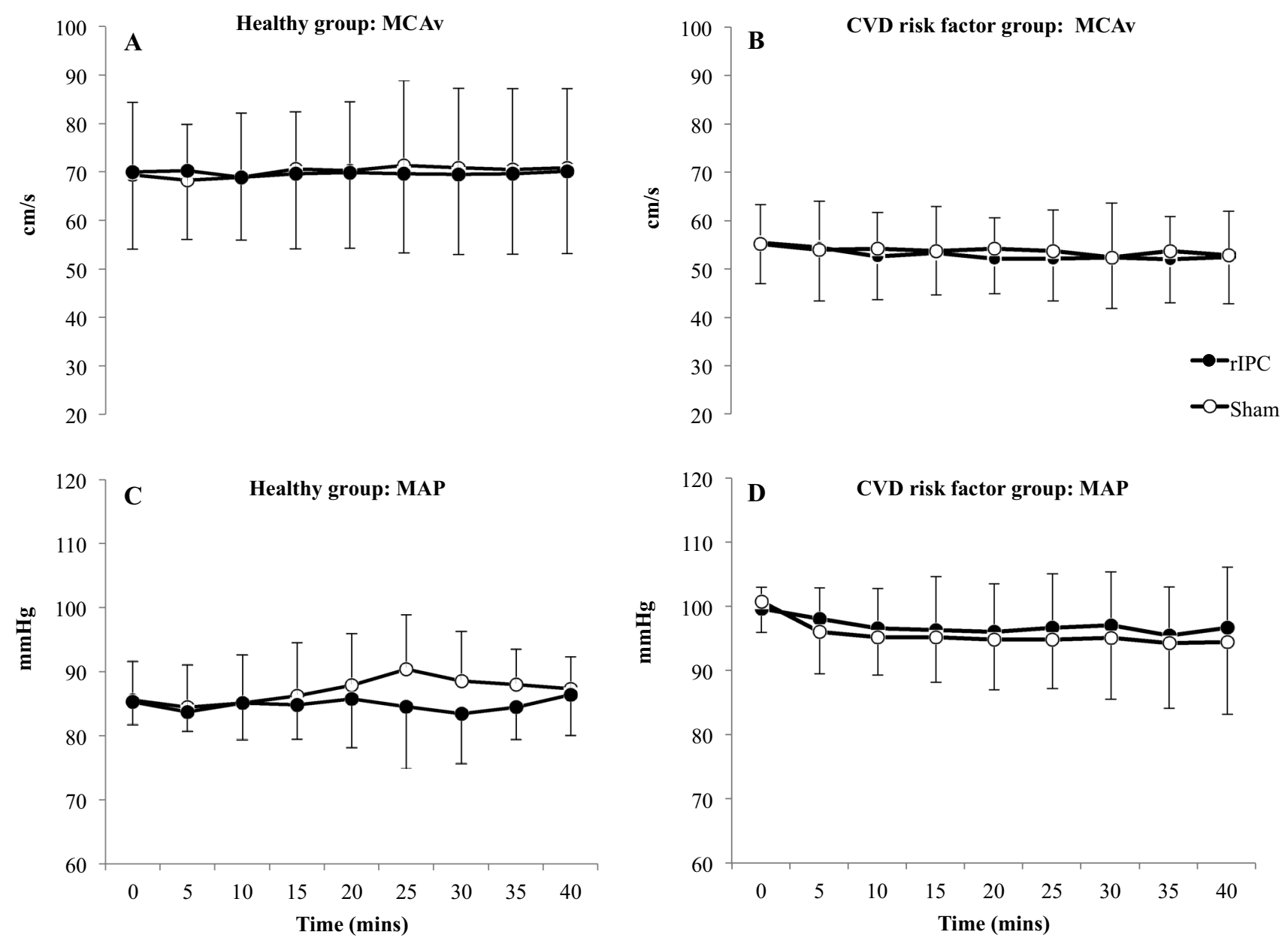

Fig. 2 Middle cerebral artery velocity (MCAv) in young healthy (a), older cardiovascular risk factor (b) individuals and mean arterial pressure (MAP) in young healthy (c) and cardiovascular risk factor (d) individuals during 40 min of RIPC and sham. Data is mean \pm SD 
Table 3 Cardiovascular and respiratory parameters during the carbon dioxide reactivity test

\begin{tabular}{|c|c|c|c|c|c|}
\hline \multirow[t]{2}{*}{ Healthy + CVD Risk } & \multicolumn{2}{|l|}{ Sham } & \multicolumn{2}{|l|}{ RIPC } & \multirow{2}{*}{$\begin{array}{l}\text { Sham v } \\
\text { RIPC } P \\
\text { value }\end{array}$} \\
\hline & Baseline & $5 \% \mathrm{CO}_{2}$ & Baseline & $5 \% \mathrm{CO}_{2}$ & \\
\hline $\operatorname{MCAv}(\mathrm{cm} / \mathrm{s})$ & $64 \pm 12$ & $83 \pm 19 *$ & $63 \pm 11$ & $83 \pm 18^{*}$ & \\
\hline $\mathrm{PetCO}_{2}(\mathrm{mmHg})$ & $34 \pm 6$ & $44 \pm 3^{*}$ & $34 \pm 6$ & $44 \pm 3^{*}$ & \\
\hline MAP (mmHg) & $101 \pm 8$ & $108 \pm 9^{*}$ & $100 \pm 5$ & $106 \pm 6^{*}$ & \\
\hline $\begin{array}{l}\text { MCA reactivity to } \mathrm{CO}_{2} \\
(\mathrm{~cm} / \mathrm{s} / \mathrm{mmHg})\end{array}$ & $1.97 \pm 0.88$ & & $2.06 \pm 0.69$ & & 0.61 \\
\hline
\end{tabular}

Values are means \pm SD. Data are presented grouped (Healthy and CVD risk) as there were no significant interactions between participant groups. Statistical significance was set at $P<0.05$

$M C A v$ middle cerebral artery velocity, $\mathrm{PetCO}_{2}$ partial pressure of end tidal carbon dioxide, MAP mean arterial pressure

* Statistically significant from baseline at $P<0.001$ both $P<0.001)$. MCAv subsequently increased, with no difference between the sham and RIPC conditions (Table 3, $P=0.43$ ). There was no overall effect of RIPC on $\mathrm{CO}_{2}$ reactivity compared to the sham condition (group $\times$ treatment $\times \mathrm{PetCO}_{2}, P=0.61$, Table 3).

\section{Impact of RIPC on normocapnic and hypercapnic cerebral autoregulation}

During normocapnia, there were no main effects or interactions in the low frequency $(0.10 \mathrm{~Hz})$ for normalised gain (Table $4, P=0.46)$, phase $(P=0.53)$ or coherence $(P=0.59)$ between the sham and RIPC conditions. PetCO $\mathrm{O}_{2}$ values during the squat-stand procedure were not different between conditions $(P=0.81)$.

Similarly, during hypercapnia, no significant main effects or interactions in the low frequency domains for normalised gain (Table $4, P=0.11)$, phase $(P=0.90)$ or coherence
( $P=0.45)$ were observed. PetCO $\mathrm{O}_{2}$ values during hypercapnia did not differ between conditions $(P=0.90)$.

\section{Effect of hypercapnia on cerebral autoregulation (comparison of sham conditions)}

Hypercapnia induced a phase reduction of 0.15 radians $(0.08,0.34)$ when compared to normocapnic cerebral autoregulation $(P=0.002)$. Additionally, normalised gain decreased during hypercapnic cerebral autoregulation by $0.41 \%(0.21,0.47)$ compared to normocapnic $(P<0.001)$.

\section{Discussion}

This is the first study to investigate the acute impact of RIPC on both dynamic cerebral autoregulation and cerebrovascular $\mathrm{CO}_{2}$ reactivity in healthy humans and those at increased risk of cardiovascular disease and stroke. Our principle

Table 4 Transfer function analysis of oscillations in mean arterial pressure and middle cerebral artery velocity using squat-stand manoeuvres

\begin{tabular}{|c|c|c|c|c|c|c|}
\hline \multirow[t]{2}{*}{ Healthy + CVD risk } & \multicolumn{3}{|c|}{ Normocapnia $(n=19 ;$ healthy $=11)$} & \multicolumn{3}{|c|}{ Hypercapnia $(n=17$; healthy $=11)$} \\
\hline & Sham & RIPC & $P$ value & Sham & RIPC & $P$ value \\
\hline $\mathrm{PetCO}_{2}(\mathrm{mmHg})$ & $38.35 \pm 2.65$ & $38.15 \pm 2.85$ & 0.81 & $46.63 \pm 2.74$ & $46.46 \pm 3.00$ & 0.90 \\
\hline \multicolumn{7}{|c|}{ Dynamic cerebral autoregulation $(0.10 \mathrm{~Hz})$} \\
\hline MCAv power $(\mathrm{cm} / \mathrm{s})^{2}$ & $81.24 \pm 54.43$ & $80.95 \pm 60.47$ & 0.87 & $76.85 \pm 40.04$ & $82.82 \pm 52.66$ & 0.69 \\
\hline MAP power $\left(\mathrm{mmHg}^{2}\right)$ & $108.83 \pm 55.61$ & $117.67 \pm 72.05$ & 0.73 & $110.81 \pm 62.25$ & $119.47 \pm 88.78$ & 0.74 \\
\hline Normalised gain $\%$ & $1.27 \pm 0.25$ & $1.22 \pm 0.35$ & 0.46 & $0.86 \pm 0.16$ & $0.94 \pm 0.21$ & 0.11 \\
\hline Gain $(\mathrm{cm} / \mathrm{s} / \mathrm{mmHg})$ & $0.80 \pm 0.17$ & $0.75 \pm 0.17$ & 0.86 & $0.75 \pm 0.16$ & $0.80 \pm 0.20$ & 0.82 \\
\hline Phase (radians) & $0.53 \pm 0.47$ & $0.64 \pm 0.39$ & 0.53 & $0.38 \pm 0.44$ & $0.40 \pm 0.31$ & 0.90 \\
\hline Coherence & $0.64 \pm 0.10$ & $0.65 \pm 0.10$ & 0.59 & $0.60 \pm 0.20$ & $0.58 \pm 0.11$ & 0.45 \\
\hline
\end{tabular}

Comparison between Sham and RIPC conditions with all participants grouped together

Values are means \pm SD. Data are presented grouped (healthy and CVD risk) as there were no significant interactions between participant groups. Statistical significance was set at $P<0.05$

$\mathrm{PetCO}_{2}$ partial pressure of end tidal carbon dioxide, $M C A v$ middle cerebral artery velocity, MAP, mean arterial pressure 
findings are (1) resting cerebral blood velocity was significantly higher at baseline in the healthy group compared to the cardiovascular risk group and (2) RIPC did not impact resting cerebral perfusion, cerebrovascular $\mathrm{CO}_{2}$ reactivity or cerebral autoregulation, in either group. These findings extend our fundamental understanding of the acute effects of RIPC in humans and reveal that a single episode of RIPC does not immediately impact cerebrovascular function in humans.

Despite the well-documented effects of RIPC on myocardial and peripheral vascular function in humans $(\mathrm{B} \emptyset t \mathrm{tker}$ et al. 2010; Thielmann et al. 2013; Davies et al. 2013; Kharbanda et al. 2002; Loukogeorgakis et al. 2005; Jones et al. 2014), the present study is the first to examine the acute impact of RIPC on cerebral blood velocity, and both cerebral autoregulation and $\mathrm{CO}_{2}$ reactivity in humans. The cerebral tests above were employed to provoke cerebral vasomotion via a number of different regulatory pathways, to better identify any specific effect RIPC may have. In response to 40 min of upper arm RIPC (4 bouts per arm, alternated), we observed no concurrent impact on cerebral blood velocity. Increases in arterial diameter and blood flow to limbs and organs (heart) regional to the limb undergoing RIPC have been previously reported during the reperfusion phases of RIPC (Enko et al. 2011; Zhou et al. 2007). Our finding that RIPC did not alter cerebral blood velocity during the bout is an important observation in this context, and suggests that RIPC does not influence blood vessel function similarly in the brain. Although it is not known what mechanism/s are responsible for the regional changes in blood flow in the previous studies during RIPC, we cannot discount the possibility that RIPC did induce a change in cerebral perfusion, and that this change was counteracted by one of the numerous cerebral blood flow regulatory mechanisms (Willie et al. 2014). However, consistent with the above finding of no change in blood flow, we observed no overall impact on cerebrovascular function. Resting cerebral autoregulation, a regulatory mechanism that maintains a constant delivery of oxygenated blood to the brain despite changes in blood pressure (Aaslid et al. 1989), was unchanged by RIPC. The second aim of this study was to temporarily disturb cerebral autoregulation via hypercapnia to determine whether RIPC could attenuate the impairment. As expected (Birch et al. 1995; Zhang et al. 1998; Panerai et al. 1999; Ainslie et al. 2008), hypercapnia reduced cerebral autoregulation phase (indicating a delayed CA response time), but did not alter absolute gain, an effect consistent with some (Ainslie et al. 2008), but not all studies (Jeong et al. 2016; Zhang et al. 1998; Panerai et al. 1999). However, in contrast to our hypothesis, RIPC did not attenuate the hypercapnia-induced impairment in phase (temporal alignment). Finally, RIPC did not impact cerebrovascular reactivity to inhalation of $5 \% \mathrm{CO}_{2}$ compared to the sham condition. To our knowledge, there is only one directly relevant study that assessed RIPC and cerebrovascular function in humans (Rieger et al. 2017). In this study the authors measured cerebral blood flow responses to acute and chronic hypoxia, and found no effect of RIPC compared to controls, findings consistent with the present study. Despite this, there is increasing evidence that repeated RIPC is neuroprotective, particularly in clinical stroke and small vessels disease patients (Meng et al. 2012; England et al. 2017; Wang et al. 2017). Meng et al. previously reported that 300 days of repeated RIPC decreased stroke recurrence and interestingly noted that cerebral perfusion was higher in the RIPC group compared to the standard care patients, potentially remedying the mismatch between perfusion and metabolism. This study raises the intriguing notion that repeated bouts of RIPC may be required to influence cerebral perfusion and function to a physiologically relevant extent. Additionally, the phenomenon of RIPCmediated protection is known to be biphasic in nature, with an immediate protective period that subsides within a few hours of application, followed by a more prolonged second protective window (1-3 days) (Koch et al. 2014). Due to the difficulties in assessing the time-course of RIPC effectiveness in humans, the vast majority of these studies have been performed in animals. Although we find this unlikely, one possible explanation for our null findings is that the cerebral measures were not performed within the initial protective phase, and that the protective windows in humans may differ to that of animals, and may also be influenced by the type, number and duration of RIPC bouts.

An important aspect to this study was assessing the impact of RIPC across a spectrum of cardiovascular health, to determine if this influenced the efficacy of RIPC. Young healthy individuals typically present with unimpaired endothelial-vascular function and as the magnitude of the RIPC effect on cerebrovascular function, if any, is unknown, it is possible that a RIPC effect would be not be observable in this population. Accordingly, we assessed the effect of RIPC in healthy individuals and those at increased cardioand cerebrovascular risk. As expected, cardiovascular risk metrics were significantly different between the groups, with the young healthy individuals displaying lower mean arterial pressure and higher resting cerebral blood flows compared to the elevated risk individuals. Nonetheless, we observed no differences in the efficacy of RIPC to improve cerebral autoregulation under normo- and hypercapnic conditions between the groups.

We acknowledge the present study is not without limitations. Middle cerebral artery blood velocity was measured using transcranial Doppler, a technique that provides a reliable surrogate for absolute cerebral blood flow providing the insonated artery diameter remains constant across and between the study conditions (Ainslie and Hoiland 2014). Although unlikely, we cannot discount the possibility that 
RIPC induced a change in middle cerebral artery diameter that impacted our measures of cerebral blood flow. Our study included a mix of males and females which may have increased variability in our cerebral responses related to sex hormones (Krause et al. 2006). Additionally, the phase of the menstrual cycle in the female participants was not controlled, however as recent studies have reported that cerebral autoregulation and cerebral vascular reactivity to carbon dioxide remains unchanged across the menstrual cycle (Favre and Serrador 2019; Peltonen et al. 2016), it is unlikely it influenced our findings. Participants were screened for overt cardiovascular risk, however were not invasively screened for the presence of proximal cerebral stenosis or carotid artery disease, which if present may have impaired the cerebral autoregulatory responses. Finally, we recruited young healthy and older participants with CVD risk factors, meaning our results cannot be generalised to clinical populations. It is possible that RIPC may have had an observable effect in participants with clinical manifestation of cardio- or cerebrovascular disease and future studies will be required to determine this.

\section{Conclusion}

The findings of this study extend our fundamental knowledge on the physiological effects of RIPC in humans by assessing for the first time the acute impact of RIPC on cerebral perfusion, cerebral autoregulation and $\mathrm{CO}_{2}$ reactivity. Although acute RIPC has been found to increase peripheral blood flow (limbs, heart), we report that this effect of RIPC does not extend to the cerebral circulation, as no change was observed in cerebral perfusion during RIPC. Additionally, RIPC did not influence cerebral function, as measured by autoregulation and cerebrovascular $\mathrm{CO}_{2}$ reactivity. With recent clinical trials showing that repeated RIPC provides neuroprotection in humans, future studies are required to determine whether repeated exposure to the RIPC stimulus is necessary to induce changes in cerebral function.

Acknowledgements This study was funded by the Danish Diabetes Academy supported by the Novo Nordisk Foundation (PO0115) and The Independent Research Fund Denmark- Medical Sciences (DFF-6110-00021).

Author contributions HHC, JDM, YH, DHJT and HJ were involved in the conception or design of the work, the acquisition, analysis or interpretation of data for the work and in drafting the work or revising it critically for important intellectual content. AT was significantly involved in the analysis or interpretation of data for the work and in drafting the work or revising it critically for important intellectual content. All authors confirm they (1) approved the final version of the manuscript, (2) agree to be accountable for all aspects of the work in ensuring that questions related to the accuracy or integrity of any part of the work are appropriately investigated and resolved, and (3) all persons designated as authors qualify for authorship, and all those who qualify for authorship are listed.

\section{Compliance with ethical standards}

Conflict of interest The author declares that there is no competing interest.

Open Access This article is licensed under a Creative Commons Attribution 4.0 International License, which permits use, sharing, adaptation, distribution and reproduction in any medium or format, as long as you give appropriate credit to the original author(s) and the source, provide a link to the Creative Commons licence, and indicate if changes were made. The images or other third party material in this article are included in the article's Creative Commons licence, unless indicated otherwise in a credit line to the material. If material is not included in the article's Creative Commons licence and your intended use is not permitted by statutory regulation or exceeds the permitted use, you will need to obtain permission directly from the copyright holder. To view a copy of this licence, visit http://creativecommons.org/licenses/by/4.0/.

\section{References}

Aaslid R, Lindegaard KF, Sorteberg W, Nornes H (1989) Cerebral autoregulation dynamics in humans. Stroke 20(1):45-52. https ://doi.org/10.1161/01.str.20.1.45

Ainslie PN, Hoiland RL (2014) Transcranial Doppler ultrasound: valid, invalid, or both? J Appl Physiol 117(10):1081-1083. https ://doi.org/10.1152/japplphysiol.00854.2014

Ainslie PN, Murrell C, Peebles K, Swart M, Skinner MA, Williams MJA, Taylor RD (2007) Early morning impairment in cerebral autoregulation and cerebrovascular $\mathrm{CO}_{2}$ reactivity in healthy humans: relation to endothelial function. Exp Physiol 92(4):769-777. https://doi.org/10.1113/expphysiol.2006.03681 4

Ainslie PN, Celi L, McGrattan K, Peebles K, Ogoh S (2008) Dynamic cerebral autoregulation and baroreflex sensitivity during modest and severe step changes in arterial $\mathrm{PCO}_{2}$. Brain Res 1230:115124. https://doi.org/10.1016/j.brainres.2008.07.048

Birch AA, Dirnhuber MJ, Hartley-Davies R, Iannotti F, Neil-Dwyer $\mathrm{G}$ (1995) Assessment of autoregulation by means of periodic changes in blood pressure. Stroke 26(5):834

Bøtker HE, Kharbanda R, Schmidt MR, Bøttcher M, Kaltoft AK, Terkelsen CJ, Munk K, Andersen NH, Hansen TM, Trautner S, Lassen JF, Christiansen EH, Krusell LR, Kristensen SD, Thuesen L, Nielsen SS, Rehling M, Sørensen HT, Redington AN, Nielsen TT (2010) Remote ischaemic conditioning before hospital admission, as a complement to angioplasty, and effect on myocardial salvage in patients with acute myocardial infarction: a randomised trial. Lancet 375(9716):727-734. https://doi.org/10.1016/S0140 $-6736(09) 62001-8$

Caldas JR, Panerai RB, Haunton VJ, Almeida JP, Ferreira GSR, Camara L, Nogueira RC, Bor-Seng-Shu E, Oliveira ML, Groehs RRV, Ferreira-Santos L, Teixeira MJ, Galas FRBG, Robinson TG, Jatene FB, Hajjar LA (2016) Cerebral blood flow autoregulation in ischemic heart failure. Am J Physiol-Reg I 312(1):R108-R113. https://doi.org/10.1152/ajpregu.00361.2016

Claassen JAHR, Levine BD, Zhang R (2009) Dynamic cerebral autoregulation during repeated squat-stand maneuvers. J Appl Physiol 106(1):153-160. https://doi.org/10.1152/japplphysi ol.90822.2008 
Claassen JAHR, Meel-van den Abeelen ASS, Simpson DM, Panerai RB (2016) Transfer function analysis of dynamic cerebral autoregulation: a white paper from the International Cerebral Autoregulation Research Network, on behalf of the international Cerebral Autoregulation Research N. J Cereb Blood Flow Metab 36(4):665-680. https://doi.org/10.1177/0271678X15626425

Cocking S, Wilson M, Nichols D, Cable N, Green D, Thijssen D, Jones $\mathrm{H}$ (2018) Is there an optimal ischemic-preconditioning dose to improve cycling performance? Int J Sport Physiol 13(3):274-282. https://doi.org/10.1123/ijspp.2017-0114

Davies WR, Brown AJ, Watson W, McCormick LM, West NEJ, Dutka DP, Hoole SP (2013) Remote ischemic preconditioning improves outcome at 6 years after elective percutaneous coronary intervention. Circ Cardiovasc Interv 6(3):246

England TJ, Hedstrom A, O'Sullivan S, Donnelly R, Barrett DA, Sarmad S, Sprigg N, Bath PM (2017) RECAST (remote ischemic conditioning after stroke trial). Stroke 48(5):1412

Enko K, Nakamura K, Yunoki K, Miyoshi T, Akagi S, Yoshida M, Toh N, Sangawa M, Nishii N, Nagase S, Kohno K, Morita H, Kusano $\mathrm{KF}$, Ito $\mathrm{H}$ (2011) Intermittent arm ischemia induces vasodilatation of the contralateral upper limb. J Physiol Sci 61(6):507. https:// doi.org/10.1007/s12576-011-0172-9

Favre ME, Serrador JM (2019) Sex differences in cerebral autoregulation are unaffected by menstrual cycle phase in young, healthy women. Am J Physiol-Heart C 316(4):H920-H933. https://doi. org/10.1152/ajpheart.00474.2018

Gonzalez N, Hamilton R, Bilgin-Freiert A, Dusick J, Vespa J, Hu X, Asgari S (2013) Cerebral hemodynamic and metabolic effects of remote ischemic preconditioning in patients with subarachnoid hemorrhage. Acta Neurochir Suppl 115:193-198

Hoiland RL, Smith KJ, Carter HH, Lewis NCS, Tymko MM, Wildfong KW, Bain AR, Green DJ, Ainslie PN (2017) Shear-mediated dilation of the internal carotid artery occurs independent of hypercapnia. Am J Physiol-Heart C 313(1):H24-H31. https://doi. org/10.1152/ajpheart.00119.2017

Jensen HA, Loukogeorgakis S, Yannopoulos F, Rimpiläinen E, Petzold A, Tuominen H, Lepola P, MacAllister RJ, Deanfield JE, Mäkelä T, Alestalo K, Kiviluoma K, Anttila V, Tsang V, Juvonen T (2011) Remote ischemic preconditioning protects the brain against injury after hypothermic circulatory arrest. Circulation 123(7):714

Jensen RV, St $\varnothing$ ttrup NB, Kristiansen SB, Bøtker HE (2012) Release of a humoral circulating cardioprotective factor by remote ischemic preconditioning is dependent on preserved neural pathways in diabetic patients. Basic Res Cardiol 107(5):285. https://doi. org/10.1007/s00395-012-0285-1

Jeong S-M, Kim S-O, DeLorey DS, Babb TG, Levine BD, Zhang R (2016) Lack of correlation between cerebral vasomotor reactivity and dynamic cerebral autoregulation during stepwise increases in inspired $\mathrm{CO}_{2}$ concentration. J Appl Physiol 120(12):1434

Jones H, Hopkins N, Bailey T, Green D, Cable N, Thijssen D (2014) Seven-day remote ischemic preconditioning improves local and systemic endothelial function and microcirculation in healthy humans. Am J Hypertens 27(7):918-925

Kharbanda RK, Mortensen UM, White PA, Kristiansen SB, Schmidt MR, Hoschtitzky JA, Vogel M, Sorensen K, Redington AN, MacAllister R (2002) Transient limb ischemia induces remote ischemic preconditioning in vivo. Circulation 106(23):2881

Koch S, Della-Morte D, Dave KR, Sacco RL, Perez-Pinzon MA (2014) Biomarkers for ischemic preconditioning: finding the responders. J Cerebr Blood Flow Metab 34(6):933-941. https://doi. org/10.1038/jcbfm.2014.42

Krause DN, Duckles SP, Pelligrino DA (2006) Influence of sex steroid hormones on cerebrovascular function. J Appl Physiol 101(4):1252-1261. https://doi.org/10.1152/japplphysiol.01095 .2005
Lavi S, Gaitini D, Milloul V, Jacob G (2006) Impaired cerebral $\mathrm{CO}_{2}$ vasoreactivity: association with endothelial dysfunction. Am J Physiol Heart C 291(4):H1856-H1861. https://doi.org/10.1152/ ajpheart.00014.2006

Lim SY, Hausenloy DJ (2012) Remote ischemic conditioning: from bench to bedside. Front Physiol 3:27. https://doi.org/10.3389/ fphys.2012.00027

Loukogeorgakis SP, Panagiotidou AT, Broadhead MW, Donald A, Deanfield JE, MacAllister RJ (2005) Remote ischemic preconditioning provides early and late protection against endothelial ischemia-reperfusion injury in humans: role of the autonomic nervous system. J Am Coll Cardiol 46(3):450-456. https://doi. org/10.1016/j.jacc.2005.04.044

Meng R, Asmaro K, Meng L, Liu Y, Ma C, Xi C, Li G, Ren C, Luo Y, Ling F, Jia J, Hua Y, Wang X, Ding Y, Lo EH, Ji X (2012) Upper limb ischemic preconditioning prevents recurrent stroke in intracranial arterial stenosis. Neurology 79(18):1853-1861

Panerai RB, Deverson ST, Mahony P, Hayes P, Evans DH (1999) Effect of $\mathrm{CO}_{2}$ on dynamic cerebral autoregulation measurement. Physiol Meas 20(3):265

Peltonen GL, Harrell JW, Aleckson BP, LaPlante KM, Crain MK, Schrage WG (2016) Cerebral blood flow regulation in women across menstrual phase: differential contribution of cyclooxygenase to basal, hypoxic, and hypercapnic vascular tone. Am J Physiol Reg I 311(2):R222-R231. https://doi.org/10.1152/ajpre gu.00106.2016

Przyklenk K, Bauer B, Ovize M, Kloner RA, Whittaker P (1993) Regional ischemic preconditioning protects remote virgin myocardium from subsequent sustained coronary occlusion. Circulation 87(3):893

Ren C, Gao X, Steinberg GK, Zhao H (2008) Limb remote-preconditioning protects against focal ischemia in rats and contradicts the dogma of therapeutic time windows for preconditioning. Neuroscience 151(4):1099-1103. https://doi.org/10.1016/j.neuroscien ce.2007.11.056

Rieger MG, Hoiland RL, Tremblay JC, Stembridge M, Bain AR, Flück D, Subedi P, Anholm JD, Ainslie PN (2017) One session of remote ischemic preconditioning does not improve vascular function in acute normobaric and chronic hypobaric hypoxia. Exp Physiol 102(9):1143-1157. https://doi.org/10.1113/EP086441

Shimizu M, Tropak M, Diaz Roberto J, Suto F, Surendra H, Kuzmin E, Li J, Gross G, Wilson Gregory J, Callahan J, Redington Andrew N (2009) Transient limb ischaemia remotely preconditions through a humoral mechanism acting directly on the myocardium: evidence suggesting cross-species protection. Clin Sci 117(5):191

Smirl JD, Hoffman K, Tzeng Y-C, Hansen A, Ainslie PN (2015) Methodological comparison of active- and passive-driven oscillations in blood pressure; implications for the assessment of cerebral pressure-flow relationships. J Appl Physiol 119(5):487-501. https ://doi.org/10.1152/japplphysiol.00264.2015

Thielmann M, Kottenberg E, Kleinbongard P, Wendt D, Gedik N, Pasa S, Price V, Tsagakis K, Neuhäuser M, Peters J, Jakob H, Heusch G (2013) Cardioprotective and prognostic effects of remote ischaemic preconditioning in patients undergoing coronary artery bypass surgery: a single-centre randomised, double-blind, controlled trial. Lancet 382(9892):597-604. https://doi.org/10.1016/ S0140-6736(13)61450-6

Wang Y, Meng R, Song H, Liu G, Hua Y, Cui D, Zheng L, Feng W, Liebeskind DS, Fisher M, Ji X (2017) Remote ischemic conditioning may improve outcomes of patients with cerebral small-vessel disease. Stroke 48(11):3064

Willie CK, Tzeng YC, Fisher JA, Ainslie PN (2014) Integrative regulation of human brain blood flow. J Physiol 592(5):841-859. https ://doi.org/10.1113/jphysiol.2013.268953 
Zhang R, Zuckerman JH, Giller CA, Levine BD (1998) Transfer function analysis of dynamic cerebral autoregulation in humans. Am J Physiol Heart C 274(1):H233

Zhou K, Yang B, Zhou X-M, Tan C-M, Zhao Y, Huang C, Liao X-B, Xiao HB (2007) Effects of remote ischemic preconditioning on the flow pattern of the left anterior descending coronary artery in normal subjects. Inter J Cardiol 122(3):250-251. https://doi. org/10.1016/j.ijcard.2006.11.079
Publisher's Note Springer Nature remains neutral with regard to jurisdictional claims in published maps and institutional affiliations. 